\title{
Use of immuno-dominant epitope derived from genotype 4 as a diagnostic reagent for detecting the antibodies against Hepatitis E Virus
}

Xiu Bing-shui ${ }^{1 \dagger}$, Feng Xiao-yan ${ }^{1 \dagger}$, He Jing $^{1}$, Chen Kun ${ }^{1}$, Liu Jing ${ }^{3}$, Dai Zhen-hua ${ }^{1}$, Yang Xi-Qin ${ }^{1}$, Wang Guo-hua ${ }^{1}$, Wang You-chun ${ }^{2}$, Zhang He-qiu ${ }^{1 *}$, Song Xiao-guo ${ }^{1}$ and Zhu Cui-xia ${ }^{1}$

\begin{abstract}
Background: Despite the genotype 4 has become the dominant cause of hepatitis $E$ disease in China, none antigen derived from genotype 4 of hepatitis E virus (HEV) was used in current commercial anti-HEV immunoassay, and the serological reactivity of antigen derive from genotype 4 is not well-charactered.

Methods: We expressed and purified the 4 main immuno-dominant epitopes derived from genotype 1 and 4 including ORF2 (410-621aa) of genotype 4, ORF3 (47-114aa) of genotype 4, ORF2 (396-606aa) of genotype 1 and ORF3 (56-123aa) of genotype 4.

Results: The ORF2 of genotype 4 displayed good diagnostics performance according to ROC analysis using in-house panel, and the immunoassays based the ORF2 of genotype 4 was then developed to detect the anti-HEV IgG antibodies and evaluated further in 530 anti-HEV IgG positive specimens and 380 negative specimens. The sensitivity and the specificity is $98.1 \%$ (520/530) and $94.7 \%$ (360/380) for immunoassay based on ORF2 of genotype 4, 96.6\% (512/530) and 92.6\% (352/380) for commercial immunoassay based on genotype 1. It is noted that all of the positive samples will be detected by combing two assays together. The anti-HEV immunoassays based on genotype 4 are in accordance with Chinese anti-HEV national standard,and show an good agreement of 95.8\% with commercial assay (kappa $=0.913, \mathrm{P}=0.014$ ).
\end{abstract}

Conclusions: The immunoassay based on ORF2G4 displays good performance, and combining assay based on genotype 1 together with genotype 4 will benefit the HEV diagnosis in large scale samples.

Keyword: HEV, ORF2, ORF3, Genotype, Immunoassay

\section{Background}

Hepatitis E caused by Hepatitis E Virus (HEV) has been reported all over the world. Usually hepatitis $E$ is endemic in developing countries associated with contaminated drinking water. In China, there are about 120,000 people infected with HEV and lead to 707 deaths in Xinjiang, during 1986-1988 [1]. In developed countries, hepatitis E occurs sporadically either related to travel to endemic areas or caused by autochthonous strains [2]. Now many animal including wild boars, deer, pig, horses, rabbits etc.,

\footnotetext{
* Correspondence: zhangheqiu2004@yahoo.com.cn

${ }^{\dagger}$ Equal contributors

'Institute of Basic Medical Sciences, Academy of military Medical Sciences, Taiping Road, Beijing 100850, P.R. China

Full list of author information is available at the end of the article
}

was found to carry the virus, which is the potential reason contributed to the transmission of HEV [3,4].

The completion of the HEV genome facilitated the development of the HEV diagnostics. The HEV genome is a single-stranded, positive-sense RNA encoding three open reading frames (ORFs) named ORF1, ORF2 and ORF3 [5]. Now recombinant ORF2 and ORF3 antigens or immunodominant peptides were widely used in commercial HEV serological test including detecting IgM, IgG, IgA antibodies against HEV [6,7]. Recently, RT-PCR is a new way to detect HEV-RNA [8]. However, in addition to high expense and laborious work of the RT-PCR, HEV RNA exists only shortly in the blood and feces among sub-clinical cases [9]. Therefore, HEV immunoassays remain important and irreplaceable in the diagnosis of HEV 
infection especially in developing countries where HEV infection is often endemic.

Now four distinct genotypes (genotypes1-4) have been identified according to the phylogenic analyze of the HEV [10]. In spite only one serotype was found, recent report revealed that there are different antigenicity of HEV ORF2 between genotype 1 and 3 [11]. It is reported that anti-HEV were not detectable in a patient infected with HEV strain US-1 using an assay based on Burmese and Mexican strains [12]. Anti-ORF3 antibodies were detected in monkey infected with genotype 1 and 2 but not in monkey infected with genotype 3 or 4 [13]. All above evidences mean the sensitivity of the HEV serological test in definite geographic area is depend on the prevailed genotype and the immunodominant antigen used in the immunoassays.

Genotype 4 is originally identified in China in 2002, and with one nucleotide insertion in ORF2 which leaded to increased 13 amino acids at its $\mathrm{C}$ terminal comparing with other genotype [14]. Our collaborated research revealed that the ORF3 polypeptide of genotype 4 displayed stronger reactivity than that of genotype 1 in the sera from monkeys infected with genotype 4 [15]. Immunoassays based on ORF2 immuno-dominant epitope derived from HEV genotype 4 detected some cases of acute hepatitis $\mathrm{E}$ undetected by a commercial assay [16]. That means the antigen derived from genotype 4 is important in diagnosis anti-HEV especially in China where the genotype 4 and 1 were prevalent in recent report $[17,18]$. But until now, no commercial assay is developed based on antigen derived from genotype 4, and little is known about the sensitivity and specificity of immunoassay based on antigen derived from genotype 4 in large random samples of patients infected with HEV.

The aim of this study is to develop the immunoassay based on recombinant immuno-dominant HEV antigen derived from genotype 4 which has never been used in commercial test. Total of 910 samples were used to evaluate the sensitivity and specificity of the new HEV immunoassay comparing with commercial immunoassay based on genotype 1 .

\section{Results}

1. Selection and expression of HEV immuno-dominant epitopes derive from genotype 4 and 1 .

The selection of the immuno-dominant antigen is the key for the development of the immunoassay. The ORF2G1 (396-606aa, genotype 1) is well-characterized as an immunoreactive antigen and widely used in commercial antiHEV immunoassay. Because the full length ORF2 from genotype 4 is 14 amino acids longer than that from genotype 1 for a single nucleotide insertion, the corresponding
ORF2 immuno-domain of genotype 4 (ORF2G4) is 410621aa. The sequences alignment revealed that there were 20 amino acids (9.52\%) different between genotype 4 and 1 (Figure 1A), which lead to a little change in hydrophobicity analyzed by the BioSun software (Figure 2).

The nucleotide insertion of genotype 4 also changes the sequences of ORF3. The ORF3 of genotype 4 is 9 amino acids shorter than that from genotype 1 at the $\mathrm{N}$ terminal. However, polypeptide at the $\mathrm{N}$ terminal of ORF3 display no immunoreactive with anti- HEV positive sera including both genotype 4 (1-60aa) and genotype 1(1-51aa) in our collaborated researches [19]. The ORF3 (56-123aa) of genotype 1 (ORF3G1) and ORF3 (47-114aa) of genotype 4 (ORF3G4) were selected instead of the whole ORF3. The sequences alignment (Figure 1B) revealed that 20aa $(29.4 \%)$ is different between he genotype 4 and 1 ORF3 selected, which lead to much change in hydrophobicity (Figure 3).

Above 4 antigens were expressed in insoluble form. Following purification, each product showed one clear band of the expected molecular mass on SDS-PAGE.

2. Reactivity of ORF2G1, ORF2G4, ORF3G1 and ORF3G4 detected by in-house panel.

The in-house panel was used to determine the reactivity of HEV by indirect ELISA including 20 anti-HEV IgG positive sera and 20 anti-HEV IgG negative sera. Both ORF2 antigens derived from genotype 1 and 4 display strong reactions with all of 20 anti-HEV positive sera. Comparably, the antigenicity of ORF3 is weaker than that of ORF2. Only 12 of 20 positive sera were reacted with ORF3. There are no sera positive with ORF3 and negative with ORF2. That means that adding ORF3 antigen could have little help to acquire high sensitivity of anti-HEV detection in the in-house panel. However, there are $2 \mathrm{HEV}$ positive sera display inconsistent results between the ORF3 antigen of genotype 1 and 4 (Table 1). Further ROC curve showed that the ORF2G4 achieved best performance with area under the curve 0.988 (Figure 4), and was selected to develop the HEV immunoassay.

3. Establish and evaluate HEV-IgG Immunoassay based on the ORF2G4 antigen.

400 plasma of health blood donor were used to determine the cut-off value of the assay. The cut-off value was 0.18 based on the average value plus triple standard deviation according to the statistical analysis. The specimen with $\mathrm{S} / \mathrm{CO}$ ratio $\geq 1.0$ is positive, the specimen with $\mathrm{S} / \mathrm{CO}$ ratio $<1.0$ is negative.

The Chinese National Reference was used to evaluate the performance of our immunoassay based on 


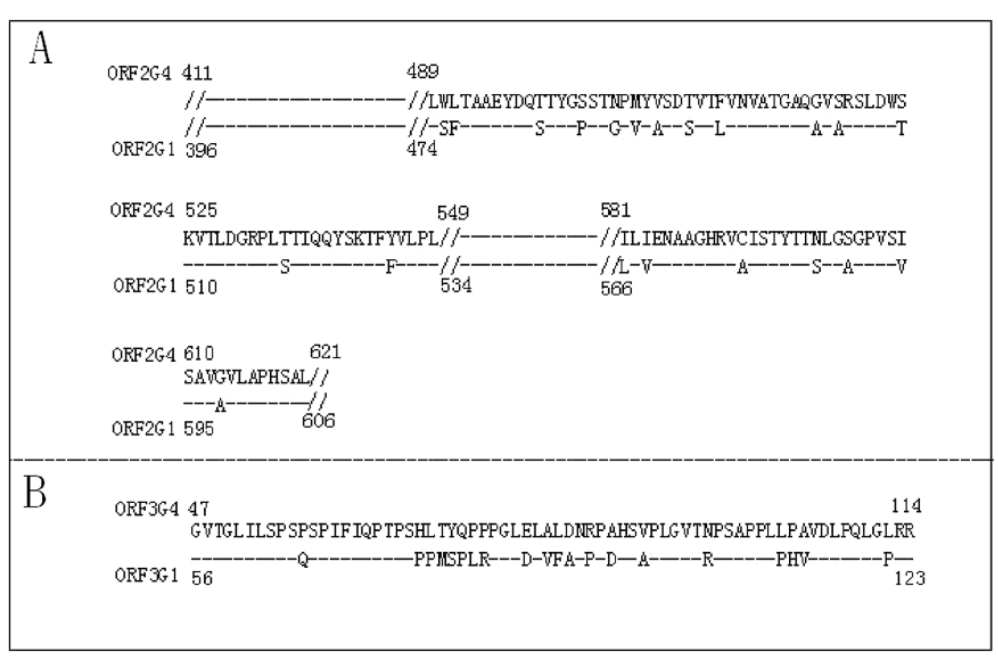

Figure 1 The alignment of the deduced sequences of the amino acids of HEV sequences. $\mathbf{A}$ is the alignment results of ORF2 between genotype 1 and 4; $\mathbf{B}$ is the alignment results of ORF3 between genotype 1 and 4; identical amino acids indicated by dashes.

genotype 4 . There are 30 true negative, 10 positive specimens, 4 sera with serial dilution, 1 precision specimens in Chinese National Reference for anti-HEV antibody detection. Our result is as follows: all of the positive and negative specimens were correctly identified. The sensitivity test using the sera with serial dilution is 1:16 and precision CV is $4 \%$. These results are consistent with the national standard.

We use a total of 910 specimens to evaluate our antiHEV immunoassay. $530 \mathrm{HEV} \mathrm{IgG} \mathrm{positive} \mathrm{samples} \mathrm{and}$ 380 negative samples (190 HBV infected sample, 190 health blood donor) were included. The results were shown in Table 2 . The sensitivity of IgG assay based on genotype 4 is $98.1 \%(520 / 530)$ in the anti-IgG positive sample, which is little higher than that of commercial immunoassay based on genotype 1 (96.6\%, 512/530) $\left(\chi^{2}=2.384, \mathrm{P}=0.125\right)$. The specificity of assay based on genotype 4 and 1 is $94.7 \%(360 / 380)$ and $92.6 \%(352 /$ 380) respectively in anti-IgG negative panel. There are no significant difference between two assays $\left(\chi^{2}=1.767\right.$, $\mathrm{P}=0.184)$.

To better understand the influence brought by different HEV genotype antigen, we analyzed the agreement between the 2 immunoassay in positive and negative samples respectively. The results were summarized in Table 3. The two tests gave an excellent agreement of

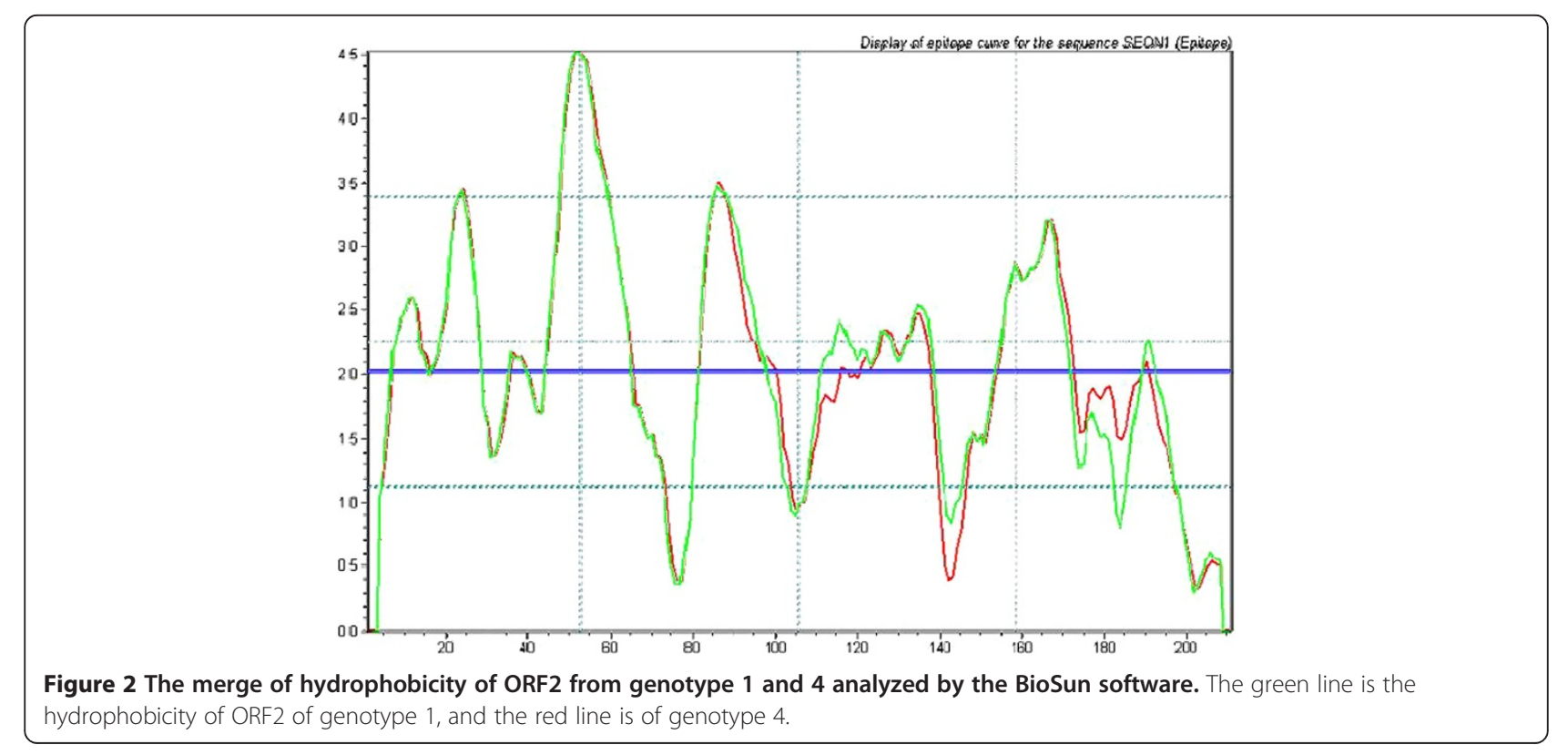




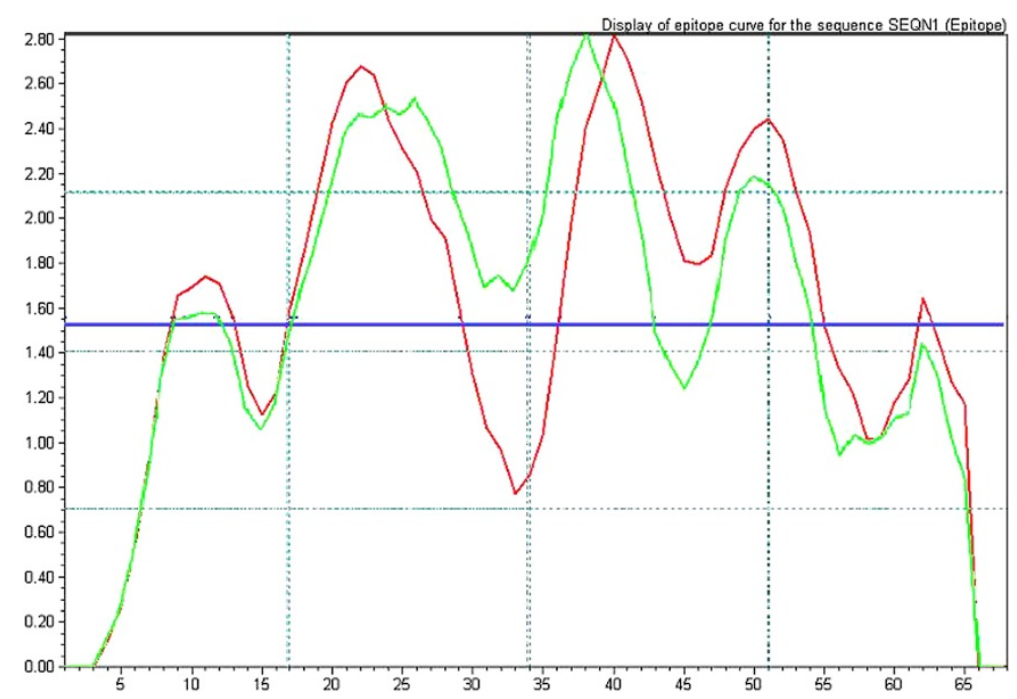

Figure 3 The merge of hydrophobicity of ORF3 from genotype 1 and 4 analyzed by the BioSun software. The green line is the hydrophobicity of ORF3 from genotype 1, and the red line is from genotype 4 .

95.8\% with a kappa statistic of $0.913(\mathrm{P}=0.014)$ in 910 samples. It is noted there is no samples negative with both test in positive panel and the detectable rates could reach $100 \%$ in 530 positive samples when combining two assays together.

\section{Discussion}

The antigen used in the immunoassay is the key to acquire high sensitivity and specificity. It was reported that ORF2 (394-606aa) of the genotype 1 expressed in E.coli contained the conformational neutralizing epitopes, and display high immuno-reactivity with both acute and convalescent sera of HEV patients [20]. But the full-length ORF2 expressed will mask some epitope, and display weak immunoreactivity in detecting anti-HEV antibodies [21]. Now, ORF2 (394-606aa) of the genotype 1 is the main antigen widely used in the diagnosis of anti-HEV antibodies [22]. However, the valuable epitopes derived from genotype 4 has not yet been well investigated. Using the conformational antibodies 1 G10 of genotype 4, ORF2 (477-613aa) was confirmed as conformationaldependent epitope, and its flanking amino acid residues is important to form the stable conformation of the epitope [23]. Our previous research found the ORF2 (384-673aa) of genotype 4 predicted by bio-information

Table 1 Comparison between the ORF3G1 and ORF3G4 by ELISA in in-house panel

\begin{tabular}{lcc}
\hline ORF3G1 & \multicolumn{2}{c}{ ORF3G4 } \\
\cline { 2 - 3 } & Positive & Negative \\
\hline Positive & 11 & 1 \\
Negative & 1 & 22 \\
\hline
\end{tabular}

comprise epitope mentioned above and displayed good reactivity with serum of infected patients and monkey inoculated with HEV experimentally. In this paper, we confirmed the results again that the ORF2 (410-621aa) of genotype 4 is immunoreactive just as ORF2 (374606aa) of genotype 1 .

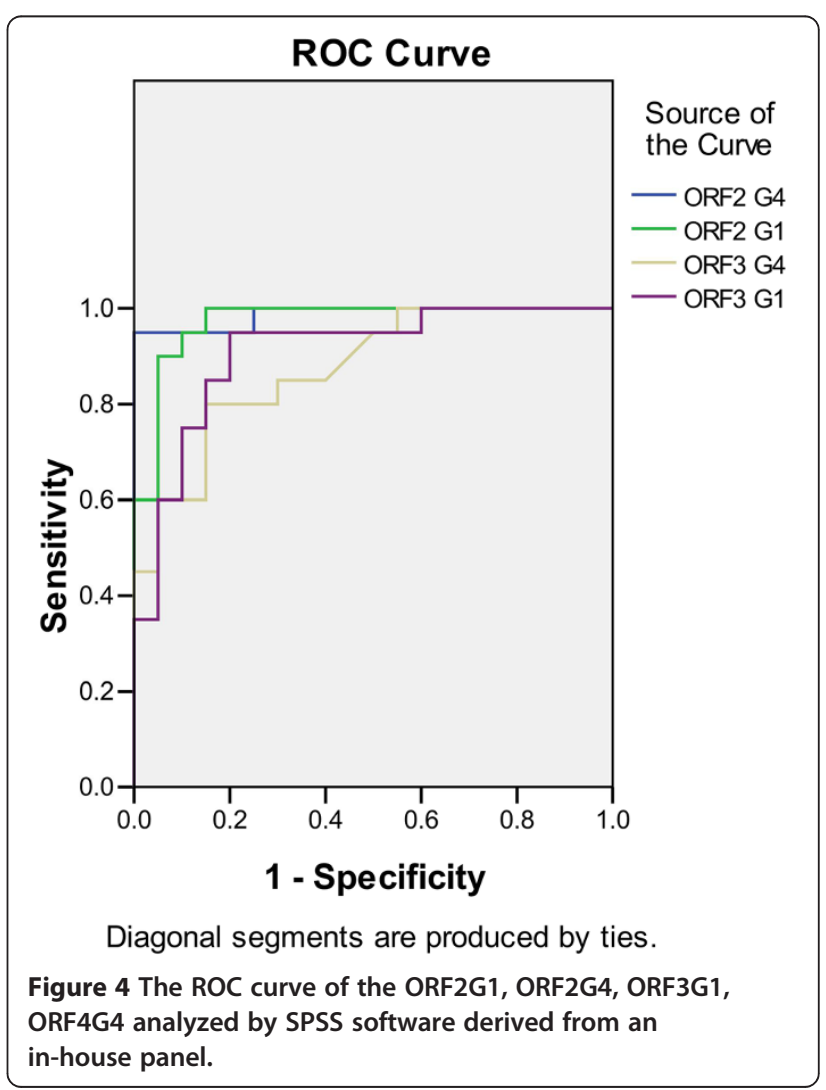


Table 2 The results of anti-HEV IgG Immunoassay based on genotype 1 and 4

\begin{tabular}{cccc}
\hline Group & Number & $\begin{array}{c}\text { positive by assay } \\
\text { based on genotype 1 }\end{array}$ & $\begin{array}{c}\text { positive by assay } \\
\text { based on genotype 4 }\end{array}$ \\
\hline $\begin{array}{c}\text { HEV IgG } \\
\text { positive }\end{array}$ & 530 & 512 & 520 \\
HBV & 190 & 15 & 9 \\
$\begin{array}{c}\text { blood } \\
\text { donor }\end{array}$ & 190 & 13 & 11 \\
\hline
\end{tabular}

It is doubtful about the diagnostics value of the ORF 3 antigen used in anti-HEV detection. Some authors reveal that the assay based on ORF3 could detect some early antibodies negative with ORF2 [24,25]. While others found the kits using both ORF3 and ORF2 did not display more sensitive than the one used ORF2 alone [26]. As samples used were from convalescent and not early infected patients, we found there were no samples with anti-ORF3 positive but anti-ORF2 negative results in this study. However, our data revealed that the ORF3 of genotype 1 and 4 displayed different reactive patterns in the in-house panel, and it is reported that the ORF3 display genotypic difference in others' researches [19]. That suggested the ORF3 antigen could be of potential use in HEV serotype detection.

Anti-IgG was mainly appear in the convalescent serum,while anti-HEV IgG also can be detected in some acute case patient [27]. That means the HEV IgG detection was used not only in epidemiology of HEV but also in diagnosis of acute HEV infection. Although there is no statistical significance, the anti-HEV assays based on genotype 4 detected little more positive samples than commercial assay did. Interestingly, some samples failed to detect in commercial assay based on genotype 1 could be detectable by the assay based on genotype 4 , and the sensitivity would be improved to $100 \%$ if we combined two assays together. There are two reason contributed for the adding effect. One is the different antigenicity of HEV ORF2 between different genotype. According to the epidemiological study,the genotype 1 and 4 are prevalent in China. That means using of antigen derived from genotype 1 and 4 will benefit the HEV detection in China. It is reported that anti-HEV were not detectable

Table 3 Agreement between immunoassay based on HEV genotype 1 and 4

\begin{tabular}{ccc}
\hline $\begin{array}{c}\text { Immunoassay based } \\
\text { on genotype 4 }\end{array}$ & \multicolumn{2}{c}{ Immunoassay based on genotype 1 } \\
\cline { 2 - 3 }+ & + & - \\
\hline- & $10+\underline{9}+\underline{19}$ & $18+\underline{1}$ \\
\hline
\end{tabular}

The normal number is the value derive from $530 \mathrm{HEV}$ positive samples, and the italic and underline number is the value derive from $380 \mathrm{HEV}$ negative samples. in a patient infected with HEV genotype 3 using an assay based on other genotype [12]. Therefore, it is necessary to develop the HEV immunoassay based on four different genotype discovered. The other reason is the various technology and procedure used by two test lead to adding affect of the diagnosis. It is well-known that the WHO recommends using 2 ELISA tests to reduce the HCV transmission by blood compounds [28]. Now, it is urged by some researchers that HEV will be transmitted by blood especially in epidemic area [29]. Our study provided the new HEV immunoassay which could complement for current commercial test.

\section{Conclusion}

In this research, we evaluated the main immuodominant epitope of HEV ORF2 and ORF3 derived respectively from genotype 4 and genotype 1 . The immunoassay based on ORF2 of genotype4 (410-621aa) was developed and displayed good performance comparing with commercial kits based on genotype1 in 910 clinical samples and blood donor. Our results reveal that combing assay based on genotype 1 together with the one based on genotype 4 will benefit the HEV diagnosis especially in large scale samples.

\section{Materials and methods Specimens}

A total of 910 specimens were used to evaluate the antiHEV immunoassay: 530 anti-HEV IgG positive specimens were from convalescent patients, 190 negative specimens were collected from healthy blood donors and 190 from HBV patients. The specimens were provided by Institute of Hepatitis, Youan Hospital, Beijing, China; Anhui Province Municipal Hospital, Hefei, China; and Shanghai public health center, Shanghai, China. 400 specimens of healthy blood donor were used for calculating the cut-off value provided by the Blood Center of Beijing Red Cross. 20 samples with anti-HEV IgG antibody positive and 20 negative samples were used as lab panel to screening the epitopes were provided by Youan Hospital. These blood and plasma specimens were stored at $-70^{\circ} \mathrm{C}$. This study was approved by the Ethics Committees of the related hospitals and research institutes.

\section{HEV antigen preparation}

Total of 4 antigens were selected in this study including ORF2 derived from genotype 1 [GenBank: M73218] (396-606aa, ORF2G1), ORF2 derived from genotype 4 [GenBank: AJ272108] (410-620aa, ORF2G4), ORF3 derived from genotype 1 [GenBank: M73218] (1-123aa, ORF3G1), ORF3 derived from genotype 4 [GenBank: AJ272108] (47-114aa, ORF3G4). The above 4 gene fragments were PCR from HEV genotype 4 or genotype 1 T-easy vector, and inserted into the pBVIL-1 vector. All of the 4 antigens 
were expressed as fusion polypeptides with an additional $17.5 \mathrm{kDa}$ of interleukin (IL)-1ßand were purified successfully using ionexchange chromatography and were then freeze-dried.

\section{Indirect ELISA to detect the HEV- IgG}

Microplates coating with $0.25 \mu \mathrm{g}$ recombinant HEV antigen were incubated in $100 \mathrm{mmol} / \mathrm{L}$ phosphate buffer (pH7.4) overnight at $4^{\circ} \mathrm{C}$, and blocked with $2 \% \mathrm{BSA}$ in the phosphate buffer at $4^{\circ} \mathrm{C}$ for $3 \mathrm{~h} .100 \mu \mathrm{L}$ of the sample were added at $1: 10$ dilution in the buffer $(100 \mathrm{mmol} / \mathrm{L}$ sodium phosphate buffer pH7.5 containing $10 \%$ goat serum and $0.05 \%$ Tween) at $37^{\circ} \mathrm{C}$ for $1 \mathrm{~h}$. the $100 \mathrm{mmol} / \mathrm{L}$ sodium phosphate buffer ( $\mathrm{pH} 7.5)$ containing $0.05 \%$ Tween were used to wash the plates five times. After incubated for 30 min at $37^{\circ} \mathrm{C}$ with 1:25000 diluted HRP-conjugated monoclonal antibodies against human $\operatorname{IgG}$, the plates were washing again. After the visualized reaction with substrate buffer $(50 \mathrm{mmol} / \mathrm{L}$ sodium phosphate-citric acid buffer, pH 5.0 containing $0.4 \mathrm{mg} / \mathrm{mL}$ TMB and $0.4 \mu \mathrm{L} / \mathrm{mL}$ of $30 \%$ hydrogen peroxide), the reaction was stopped by $50 \mu \mathrm{L}$ of $2 \mathrm{~mol} / \mathrm{L}$ sulfuric acid, and the OD450 was measured in a microplate ELISA reader. The cut-off value was calculated according to method 2.4.

\section{The evaluation of the immunoassay with the China National anti-HEV Panel}

The China national anti-HEV panel was used to assess anti-IgG HEV immunoassays based on ORF2G4. The panel included 30 true negative, 10 positive specimens, 1 specimens with dilution at $1: 4,1: 8,1: 16,1: 32$, and 1 precision specimens repetitively tested by 10 time. The standards of the China National anti-HEV IgG Panel were as follows: negative ratio is equal or more than $29 / 30$, and the positive ratio is 10/10; the sensitivity test is more than $1 / 16$ and precision $\mathrm{CV}$ is lower than $15 \%(\mathrm{n}=10)$.

\section{Comparison of the immunoassay with other commercial HEV ELISA kits}

The anti-HEV IgG antibody detection kit (indirect assay, Wan Tai Pharmaceutical Co., Lot number: EG 20090704, Xiamen, China) based on ORF2 of genotype 1 was used to evaluated the HEV antibodies assay based on HEV ORF2 of genotype 4. The above assays were performed according to the manufacturer's instructions.

\section{Statistics}

All data were analyzed by statistical software SPSS 16.0. Receiver operator characteristic curve (ROC) was conducted to evaluate the diagnostic significance of recAg-1-12. Kappa test was conducted to evaluate the consistency of qualitative results. $\mathrm{P}<0.05$ was considered statistically significant.

\section{Abbreviations}

HEV: Hepatitis E virus; ORF: The open reading frame; ORF2G4: The ORF2 of genotype 4; ORF3G4: The ORF2 of genotype 4; ORF2G1: The ORF2 of genotype 1; ORF3G1: The ORF3 of genotype 1.

\section{Competing interests}

The authors declare that they have no competing interest.

\section{Authors' contributions}

XB-s and FX-y carried out the molecular genetic and bioinformatics studies $\mathrm{HJ}$ and CK carried out the immunoassays. $\amalg$ and DZ-h participated in the sequence alignment. YX-c and WG-h purified the recombinant antigen. SX-g, ZC-X carried the SAS-PAGE. WY-C and ZH-q participated in the design of the study and coordination and helped to draft the manuscript. All authors read and approved the final manuscript.

\section{Acknowledgements}

The study was funded by the National High-Tech Science Foundation of China (Grants No.2008AA02Z434); the National Key Technologies Research and Development Program of China during the 12 Five-Year Period (Grants NO. 2011ZX10004-001)

\section{Author details}

'Institute of Basic Medical Sciences, Academy of military Medical Sciences, Taiping Road, Beijing 100850, P.R. China. ${ }^{2}$ Department of Cell Biology, National Institute for the Control of Pharmaceutical and Biological Products, No. 2 Tiantanxili, Beijing 100050, P.R. China. ${ }^{3}$ China National Center For Biotechnology Development, No. 6 West Fourth Ring Road, Beijing 100036, P.R. China.

Received: 29 June 2012 Accepted: 18 April 2013

Published: 25 April 2013

\section{References}

1. Aye TT, Uchida T, Ma XZ, Lida F, Shikata T, Zhuang H, Win KM: Complete nucleotide sequence of a hepatitis $E$ virus isolated from the Xinjiang epidemic (1986-1988) of China. Nucleic Acids Res 1992, 20:3512-3512.

2. Aggarwal R, Naik S: Epidemiology of hepatitis E: current status. J Gastroenterol Hepatol 2009, 24(9):1484-1493.

3. Geng J, Wang L, Wang X, Fu H, Bu Q, Liu P, Zhu Y, Wang M, Sui Y, Zhuang $\mathrm{H}$ : Potential risk of zoonotic transmission from young swine to human: seroepidemiological and genetic characterization of hepatitis $E$ virus in human and various animals in Beijing. China. J Viral Hepat 2011, 18(10): e583-e590.

4. Geng J, Wang L, Wang X, Fu H, Bu Q, Zhu Y, Zhuang H: Study on prevalence and genotype of hepatitis $E$ virus isolated from Rex Rabbits in Beijing, China. J Viral Hepat 2011, 18(9):661-667.

5. Reyes GR, Huang CC, Yarbough PO, Tam AW: Hepatitis E virus. Comparison of 'New and Old World' isolates. J Hepatol 1991, 13 Suppl 4:S155-S161.

6. Takahashi M, Kusakai S, Mizuo H, Suzuki K, Fujimura K, Masuko K, Sugai Y, Aikawa T, Nishizawa T, Okamoto $\mathrm{H}$ : Simultaneous detection of immunoglobulin $\mathrm{A}(\lg \mathrm{A})$ and $\lg \mathrm{M}$ antibodies against hepatitis $\mathrm{E}$ virus (HEV) Is highly specific for diagnosis of acute HEV infection. J Clin Microbiol 2005, 43(1):49-56.

7. Zhang S, Tian D, Zhang Z, Xiong J, Yuan Q, Ge S, Zhang J, Xia N: Clinical significance of anti-HEV IgA in diagnosis of acute genotype 4 hepatitis $E$ virus infection negative for anti-HEV IgM. Dig Dis Sci 2009, 54(11):2512-2518.

8. Wu KT, Chung KM, Feng IC, Sheu MJ, Kuo HT, Koay LB, Lin CY, Tang LY, Tsai SL: Acute hepatitis E virus infection in Taiwan 2002-2006 revisited: PCR shows frequent co-infection with multiple hepatitis viruses. J Med Virol 2009, 81(10):1734-1742.

9. Aggarwal R, Kini D, Sofat S, Naik SR, Krawczynski K: Duration of viraemia and faecal viral excretion in acute hepatitis E. Lancet 2000, 356(9235):1081-1082

10. Purcell $\mathrm{RH}$, Emerson SU: Hepatitis E: an emerging awareness of an old disease. J Hepatol 2008, 48(3):494-503.

11. Schlauder GG, Mushahwar IK: Genetic heterogeneity of hepatitis E virus. J Med Virol 2001, 65(2):282-292.

12. Jiménez De Oya N, Galindo I, Gironés O, Duizer E, Escribano JM, Saiz JC: Serological immunoassay for detection of hepatitis $\mathrm{E}$ virus on the basis of genotype 3 open reading frame 2 recombinant proteins produced in Trichoplusia ni larvae. J Clin Microbiol 2009, 47(10):3276-3282. 
13. Zhou YH, Purcell RH, Emerson SU: A truncated ORF2 protein contains the most immunogenic site on ORF2: antibody responses to non-vaccine sequences following challenge of vaccinated and non-vaccinated macaques with hepatitis E virus. Vaccine 2005, 23(24):3157-3165.

14. Wang $Y$, Zhang H, Ling R, Li H, Harrison TJ: The complete sequence of hepatitis $E$ virus genotype 4 reveals an alternative strategy for translation of open reading frames 2 and 3. J Gen Virol 2000, 81(Pt 7):1675-1686.

15. Ma $H$, Song $X$, Harrison $T J$, Zhang $H$, Huang $W$, Wang $Y$ : Hepatitis E virus ORF3 antigens derived from genotype 1 and 4 viruses are detected with varying efficiencies by an anti-HEV enzyme immunoassay. J Med Virol 2011, 83(5):827-832.

16. Wang Y, Zhang H, Li Z, Gu W, Lan H, Hao W, Ling R, Li H, Harrison TJ: Detection of sporadic cases of hepatitis E virus (HEV) infection in China using immunoassays based on recombinant open reading frame 2 and 3 polypeptides from HEV genotype 4. J Clin Microbiol 2001, 39(12):4370-4379.

17. Dong C, Dai X, Shao JS, Hu K, Meng JH: Identification of genetic diversity of hepatitis $E$ virus $(\mathrm{HEV})$ and determination of the seroprevalence of HEV in eastern China. Arch Virol 2007, 152(4):739-746.

18. Lam WY, Chan RC, Sung JJ, Chan PK: Genotype distribution and sequence variation of hepatitis E virus, Hong Kong. Emerg Infect Dis 2009, 15(5):792-794.

19. Ma H, Song X, Li Z, Harrison TJ, Zhang H, Huang W, Hao W, Kong W, Wang $Y$ : Varying abilities of recombinant polypeptides from different regions of hepatitis E virus ORF2 and ORF3 to detect anti-HEV immunoglobulin M. J Med Virol 2009, 81(6):1052-1061.

20. Li F, Zhuang H, Kolivas S, Locarnini SA, Anderson DA: Persistent and transient antibody responses to hepatitis $\mathrm{E}$ virus detected by western immunoblot using open reading frame 2 and 3 and glutathione S-transferase fusion proteins. J Clin Microbiol 1994, 32(9):2060-2066.

21. Li F, Torresi J, Locarnini SA, Zhuang H, Zhu W, Guo X, Anderson DA: Aminoterminal epitopes are exposed when full-length open reading frame 2 of hepatitis E virus is expressed in Escherichia coli, but carboxy-terminal epitopes are masked. J Med Virol 1997, 52(3):289-300.

22. Hu WP, Lu Y, Precioso NA, Chen HY, Howard T, Anderson D, Guan M: Double-antigen enzyme-linked immunosorbent assay for detection of hepatitis $\mathrm{E}$ virus-specific antibodies in human or swine sera. Clin Vaccine Immunol 2008, 15(8):1151-1157.

23. Zhang H, Dai X, Shan X, Meng J: The Leu477 and Leu613 of ORF2-encoded protein are critical in forming neutralization antigenic epitope of hepatitis E virus genotype 4. Cell Mol Immunol 2008, 5(6):447-456.

24. Bendall $\mathrm{R}$, Ellis V, ljaz S, Ali R, Dalton H: A comparison of two commercially available anti-HEV IgG kits and a re-evaluation of anti-HEV IgG seroprevalence data in developed countries. J Med Virol 2010, 82(5):799-805.

25. Zhang JZ, Im SW, Lau SH, Chau TN, Lai ST, Ng SP, Peiris M, Tse C, Ng TK, $\mathrm{Ng} \mathrm{MH:} \mathrm{Occurrence} \mathrm{of} \mathrm{hepatitis} \mathrm{E} \mathrm{virus} \mathrm{lgM,} \mathrm{low} \mathrm{avidity} \mathrm{lgG} \mathrm{serum}$ antibodies, and viremia in sporadic cases of non- $A,-B$, and $-C$ acute hepatitis. J Med Virol 2002, 66(1):40-48.

26. Ghabrah TM, Tsarev S, Yarbough PO, Emerson SU, Strickland GT, Purcell RH: Comparison of tests for antibody to hepatitis E virus. J Med Virol 1998, 55(2):134-137.

27. Huang S, Zhang X, Jiang H, Yan Q, Ai X, Wang Y, Cai J, Jiang L, Wu T, Wang Z, Guan L, Shih JW, Ng MH, Zhu F, Zhang J, Xia N: Profile of acute infectious markers in sporadic hepatitis E. PLoS One 2010, 5(10):e13560.

28. Safe Blood Donation. In Safe Blood and Blood products: Identifying low risk donors. WHO Module 2: Anonymous; 2002:pp. 83-pp. 84.

29. Khuroo MS, Kamili S, Yattoo GN: Hepatitis E virus infection may be transmitted through blood transfusions in an endemic area. J Gastroenterol Hepatol 2004, 19(7):778-784.

doi:10.1186/1743-422X-10-131

Cite this article as: Bing-shui et al:: Use of immuno-dominant epitope derived from genotype 4 as a diagnostic reagent for detecting the antibodies against Hepatitis E Virus. Virology Journal 2013 10:131.

\section{Submit your next manuscript to BioMed Central and take full advantage of:}

- Convenient online submission

- Thorough peer review

- No space constraints or color figure charges

- Immediate publication on acceptance

- Inclusion in PubMed, CAS, Scopus and Google Scholar

- Research which is freely available for redistribution

Submit your manuscript at www.biomedcentral.com/submit
Ciomed Central 